\title{
Ultrasound and operative evaluation of arthritic shoulder joints
}

\author{
E Alasaarela, J Leppilahti, M Hakala
}

\begin{abstract}
Objective-To assess the diagnostic value of ultrasonography (US) in the evaluation of arthritic shoulder joints.

Methods-Twenty shoulders of 20 inpatients with arthritis were evaluated by US one day before the shoulder operation. Changes in the subacromial-subdeltoid bursa, biceps tendon and tendon sheath, rotatof cuff, and glenohumeral joint were recorded and compared with findings at operation.

Results-In the detection of effusion/ hypertrophy in the subacromial-subdeltoid bursa, US had a sensitivity of $93 \%$ and a specificity of $83 \%$. For a biceps tendon rupture US had a sensitivity of $70 \%$ and a specificity of $100 \%$. US missed three intraarticular biceps tendon ruptures. For effusion/hypertrophy in the biceps tendon sheath US had a sensitivity of $100 \%$ and a specificity of $83 \%$. For a rotator cuff tear US had a sensitivity of $83 \%$ and a specificity of $57 \%$. US missed two small longitudinal rotator cuff tears. Three thin membranous, but intact, rotator cuff tendons were classified as full thickness tears by US. Synovial effusion/hypertrophy was detected by US and at operation in all of the 12 glenohumeral joints that were evaluable at surgery.

Conclusion-US is a reliable method in experienced hands for the evaluation of inflammatory changes of an arthritic shoulder. In advanced stages of rheumatoid shoulder joints, however, US is not useful, because destructive bone changes and tendon ruptures change the normal anatomy and restrict shoulder motions, limiting the visibility of US.

(Ann Rheum Dis 1998;57:357-360)
\end{abstract}

Division of

Rheumatology,

Department of

Internal Medicine

E Alasaarela

M Hakala

Department of

Surgery

J Leppilahti

University of Oulu, Finland

Correspondence to: Dr E Alasaarela, Division of Rheumatology, Department of Internal Medicine,

University of Oulu,

FIN-90220, Oulu, Finland.

Accepted for publication 17 April 1998
Although the shoulder joint seems to become involved later than the other upper limb joints in RA, the shoulder has been found to be involved already during the first two years in nearly $50 \%$ and during 14 years in $83 \%$ of patients.

Clinically, shoulder joint arthritis may often go unrecognised because of several reasons: the onset of the disease in the shoulder is often insidious, the shoulder joint is deeply seated, and synovial tissue swelling is difficult to detect. ${ }^{4}$ Plain radiography depicts rheumatoid involvement late. Ultrasonography (US) can reveal early soft tissue changes in the shoulder, such as effusions in the SA-SD bursa, ${ }^{56}$ biceps tendon sheath (BTS), ${ }^{7}$ and GH joint, ${ }^{89}$ and can also show bone erosions on the humeral head. ${ }^{10}$

The purpose of this study was to evaluate the ability of US to detect shoulder abnormalities in patients with chronic arthritis and to compare the information thus gained with the operative findings. To the best of our knowledge, this is the first study comparing preoperative US findings of arthritic shoulders with operative findings.

\section{Methods}

Twenty shoulders of 20 inpatients with chronic arthritis were evaluated by US one day before the shoulder operation. Fourteen patients were female and six were male. Their mean age was 57 (range 26-77) years. Sixteen patients had $\mathrm{RA},{ }^{11} 12$ being seropositive and four seronegative, three had ankylosing spondylitis, ${ }^{12}$ and one had psoriatic arthritis. ${ }^{13}$ The mean duration of the disease was 10 (range 1-18) years and the mean duration of the shoulder symptoms was four years (range 4 months-10 years).

The US examination was made by the author (EA), who has special training in shoulder sonography. A 7.5-MHz linear-array transducer (Aloka 2000, Tokyo, Japan) was used. Standard techniques were used for static and dynamic longitudinal and transverse scanning of the rotator cuff (RC), biceps tendon, BTS and SA-SD bursa. ${ }^{514}$ Synovial effusion/ hypertrophy of the GH joint was evaluated using the techniques reported in earlier studies. ${ }^{915}$ The sonographic findings were recorded on a data sheet during real time imaging and documented on high density printing paper with a thermal printer.

The following criteria for the classification of the US findings were used: bursal thickness $>2$ $\mathrm{mm}$ or effusion were considered as effusion/ hypertrophy of the SA-SD bursa, a hypoechoic zone completely surrounding the biceps tendon and thickening with an irregular surface of the BTS as effusion/hypertrophy of the tendon sheath. Changes in the RC were classified as a full thickness tear, a partial thickness tear or a 
Table 1 US and operative findings, diagnoses, and types of surgery for the 20 patients

\begin{tabular}{|c|c|c|c|c|c|c|c|c|c|c|c|}
\hline \multicolumn{2}{|c|}{ Bursal E/H } & \multicolumn{2}{|c|}{ Biceps tendon } & \multicolumn{2}{|c|}{$B T S E / H$} & \multicolumn{2}{|c|}{ Rotator cuff } & \multicolumn{2}{|c|}{$G H$ joint $E / H$} & \multirow[b]{2}{*}{$D G$} & \multirow[b]{2}{*}{ Type of surgery } \\
\hline US & operation & US & operation & US & operation & US & operation & US & operation & & \\
\hline yes & yes & normal & normal & yes & yes & FT tear & FT tear & yes & NA & $\mathrm{RA}+$ & BE,TSE,SRCT \\
\hline no & no & thinned & thinned & yes & yes & normal & thinned & yes & NA & RA+ & APL,TSE \\
\hline yes & yes & rupture & rupture & yes & yes & FT tear & FT tear & yes & yes & RA+ & $\mathrm{HA}$ \\
\hline yes & yes & normal & NA & no & NA & normal & FT tear* & no & NA & RA- & BE,APL,SRCT \\
\hline yes & yes & thinned & rupture & yes & NA & PT tear & PT tear* & yes & yes & RA+ & ASC,SE,APL,SRCT \\
\hline no & yes/H & thinned & thinned & yes & yes & thinned & FT tear* & yes & yes & RA+ & ASC,SE,APL,SRCT \\
\hline yes & yes & thinned & rupture & no & no & FT tear & FT tear & yes & NA & RA+ & APL,BE,RDC \\
\hline no & no & thinned & rupture & yes & yes & FT tear & membranous & yes & yes & RA+ & $\mathrm{HA}$ \\
\hline yes & yes & rupture & rupture & no & no & FT tear & membranous & yes & yes & RA+ & $\mathrm{HA}$ \\
\hline no & no & normal & normal & yes & no & FT tear & FT tear & yes & yes & RA- & ReSRCT,EXPL \\
\hline yes & yes & normal & normal & yes & yes & FT tear & FT tear & yes & yes & RA+ & APL,SRCT \\
\hline yes & yes & normal & normal & yes & yes & thickened & normal & yes & NA & AS & $\mathrm{APL}, \mathrm{BE}$ \\
\hline yes & yes & normal & normal & yes & NA & FT tear & FT tear & no & NA & RA- & APL,SRCT \\
\hline yes & yes & normal & normal & yes & yes & thinned & NA & no & NA & AS & $\mathrm{BE}, \mathrm{DCAL}$ \\
\hline yes & yes & rupture & rupture & yes & NA & FT tear & FT tear & yes & yes & RA+ & HA \\
\hline no & no & rupture & rupture & no & no & FT tear & membranous & yes & yes & RA+ & TJRA \\
\hline yes & yes & normal & normal & yes & yes & normal & normal & yes & yes & PSA & SE,TSE,APL \\
\hline no & no & rupture & rupture & no & no & FT tear & FT tear & yes & yes & RA+ & HA \\
\hline yes/H & no & rupture & rupture & yes & yes & thinned & thinned & yes & yes & RA- & HA \\
\hline yes & yes & rupture & rupture & no & no & FT tear & FT tear & yes & NA & AS & APL,SRCT \\
\hline
\end{tabular}

$\mathrm{E}=$ effusion, $\mathrm{H}=$ hypertrophy, BTS = biceps tendon sheath, $\mathrm{GH}=$ glenohumeral, $\mathrm{DG}=$ diagnoses, NA = not applicable, FT = full thickness, PT = partial thickness, $\star=$ small longitudinal tear, RA $+=$ seropositive rheumatoid arthritis, RA $-=$ seronegative rheumatoid arthritis, AS $=$ ankylosing spondylitis, PSA $=$ psoriatic arthritis, $\mathrm{BE}=$ bursectomy, TSE $=$ tenosynovectomy of the biceps tendon sheath, SRCT $=$ suturation of RC tear, APL $=$ acromioplasty, $\mathrm{HA}=$ hemiarthroplasty, ASC = arthroscopy, $\mathrm{SE}=$ synovectomy, $\mathrm{RDC}=$ resection of the distal clavicle, ReSRCT $=$ resuturation of $\mathrm{RC}$ tear, EXPL $=$ exploration, DCAL $=$ discision of the coracoacromial ligament, TJRA = total joint replacement arthroplasty.

thinned or thickened tendon. A full thickness tear was diagnosed when a defect (hypoechoic zone) extended through the tendon substance, when there was focal thinning with visible margins of the tear, or when there was a complete loss of tendon substance. A partial thickness tear was recorded when the echostructure of the tendon was heterogeneous with mixed hyperechoic and hypoechoic regions. The RC tendon was considered to be thinned when the thickness of the supraspinatus tendon (measured $2 \mathrm{~cm}$ proximal to the insertion of the distal supraspinatus) was $<3.5 \mathrm{~mm}$, and thickened when it was $>8.5 \mathrm{~mm}$; the normal thickness is $6 \mathrm{~mm}$ with a standard deviation of $1.1 \mathrm{~mm} .{ }^{16}$ Effusion/synovial hypertrophy of the GH joint was evaluated on the posterior sagittal imaging plane perpendicular to the humeral head, and in the axilla, using the criteria presented in the earlier studies. ${ }^{9} 15$ When the ultrasonographic distance between the head of the humerus and the joint capsule was $>3.0$ $\mathrm{mm}$ in the axilla or on the posterior scan, it was considered as effusion/synovial hypertrophy of the $\mathrm{GH}$ joint.

The following types of surgical operations were made: bursectomy, tenosynovectomy of the BTS, suturation or resuturation of the RC tear, acromioplasty, hemiarthroplasty, arthroscopic synovectomy, open synovectomy, discision of the coraco acromial ligament, exploration, total joint replacement arthroplasty. Not all of the shoulder structures were evaluable at every operation. The operative findings were documented on the patient's records and data sheet. We used the operative findings as a "gold standard".

Sensitivity was calculated by dividing the number of true positive results by the total number of true positive and false negative results. Specificity was calculated by dividing the number of true negative results by the total number of true negative and false positive results. Accuracy was calculated by dividing the total number of true positive and true
Table 2 Percentage validity of diagnoses by US for the 20 patients

\begin{tabular}{lllll}
\hline Validity & $\begin{array}{l}\text { Bursa E/H } \\
\left(n=20^{\star}\right)\end{array}$ & $\begin{array}{l}\text { BT rupture } \\
\left(n=19^{\star}\right)\end{array}$ & $\begin{array}{l}\text { BTS E/H } \\
\left(n=16^{\star}\right)\end{array}$ & $\begin{array}{l}\text { RC rupture } \\
\left(n=19^{\star}\right)\end{array}$ \\
\hline Sensitivity & 93 & 70 & 100 & 83 \\
Specificity & 83 & 100 & 83 & 57 \\
Accuracy & 90 & 84 & 94 & 74 \\
PVPT & 93 & 100 & 91 & 77 \\
PVNT & 83 & 75 & 100 & 67 \\
\hline
\end{tabular}

$\mathrm{BT}=$ biceps tendon, $\mathrm{PVPT}=$ predictive value of positive test PVNT $=$ predictive value of negative test, ${ }^{\star}=$ the number of patients in whom the diagnosis was confirmed at operation. Other abbreviations as table 1 .

negative results by total number of results. The predictive value of positive test was calculated by dividing the number of true positive results by the total number of true positive and false positive results. The predictive value of a negative test was calculated by dividing the number of true negative results by the total number of true negative and false negative results.

\section{Results}

In the detection of effusion/hypertrophy in the SA-SD bursa, US had a sensitivity of $93 \%$, a specificity of $83 \%$, an accuracy of $90 \%$, a predictive value of positive test of $93 \%$, and a predictive value of negative test of $83 \%$. In two cases, a mild synovial thickening of the SA-SD bursa classified as synovial hypertrophy without effusion detected by one method (US or surgery) was not confirmed by the other (table 1 and table 2). Table 2 shows the number of patients in whom the shoulder structure in question was evaluable at operation.

In the assessment of biceps tendon ruptures, US had a sensitivity of $70 \%$, a specificity of $100 \%$, an accuracy of $84 \%$, a predictive value of positive test of $100 \%$, and a predictive value of negative test of $75 \%$. US missed three intraarticular biceps tendon ruptures. In the detection of effusion/hypertrophy in the biceps tendon sheath, US had a sensitivity of $100 \%$, a specificity of $83 \%$, an accuracy of $94 \%$, a predictive value of positive test of $91 \%$, and a predictive value of negative test of $100 \%$. 
In the assessment of RC tears, US had a sensitivity of $83 \%$, a specificity of $57 \%$, an accuracy of $74 \%$, and a predictive value of positive test of $77 \%$, and a predictive value of negative test of $67 \%$. US missed one small longitudinal partial thickness tear and one small longitudinal full thickness tear. In three cases, thin membranous, but intact, RC tendons reported at surgery were classified as full thickness tears by US because of the loss of tendon substance. One RC tendon normal by US was classified as thinned at surgery, and one normal RC tendon at surgery was evaluated as thickened by US.

Synovial effusion/hypertrophy was detected by US and at surgery in all the $12 \mathrm{GH}$ joints that were evaluable at surgery. Thus US had a sensitivity of $100 \%$, but its specificity, accuracy, and predictive value of negative test were not assessable.

\section{Discussion}

This study shows that US had a high sensitivity in the detection of synovial effusion/ hypertrophy in the SA-SD bursa, BTS, and $\mathrm{GH}$ joint, whereas in the assessment of biceps and RC tendons the sensitivity and accuracy of US were lower.

Although operation can be considered the "gold standard" of the investigation methods of the shoulder, it also has some limitations. Firstly, depending on the procedure, not all structures are visible, and blind palpation of the structures does not yield exact information. Secondly, the assessments of the quality and quantity of tissues are subjective without histological tissue samples or distinct measuring instruments.

In RA, the ruptured biceps tendon is usually adherent to the bicipital groove. ${ }^{17}$ In this study, we found that in advanced stages of RA, the intra-articular tendon area was not evaluable by US because of the upward subluxation of the humeral head. In three cases where US missed intra-articular biceps tendon ruptures, the tendons appeared to be in the groove in US.

Effusion in the BTS is non-specific finding and may reflect a pathological process elsewhere in the shoulder joint. ${ }^{7}$ As the BTS is merely an extension of the synovial membrane of the joint, generalised joint effusion in arthritis can lead to fluid in the sheath. ${ }^{18}$ Effusion caused by isolated biceps tendinitis is rare. ${ }^{19}$ The BTSs of 16 shoulders were evaluable at surgery; effusion/hypertrophy in the BTS was identified in 10 of these shoulders by both US and operation. Of these 10 shoulders nine had RC abnormalities and in nine shoulders concomitant effusion/hypertrophy in the GH joint was visualised by US. Some cases with a RC rupture had no effusion/hypertrophy either in the BTS or in the GH joint. In one shoulder US depicted distinct effusion around the biceps tendon, but at operation (resuturation of the RC) no effusion was seen after incision into the subacromial space, when synovial fluid had already poured out.

The diagnostic accuracy of US in detecting RC tears seems to be controversial. ${ }^{20-23}$ US has some limitations in the evaluation of the RC. Sonograms can depict only the distal part of the RC, which is not obscured by the acromion. ${ }^{14}$ Some RC tears may not be of sufficient size or not have an appropriate configuration or differential echogenity to be detected by US. Thus, a small longitudinal tear without any retraction of the torn edges or any focal alteration in RC echogenity may not be revealed by US. ${ }^{22}$ In this study, we missed two such small longitudinal RC tears by US. Conversely, three US classified full thickness RC tears were intact at surgery, but very thin and membranous. The $\mathrm{GH}$ joints of these three shoulders were totally destroyed with the humeral heads migrated proximally and medially, greatly limiting the visualisation of the RC in US. Kelly has described three patterns of the disease in a rheumatoid shoulder. ${ }^{24}$ The most severe group comprises large erosions of the $A C$ and $\mathrm{GH}$ joints with a significant loss of glenoid bone stock and medialisation of the shoulder. The RC may be thinned, but is intact in at least $80 \%$ of these patients. On the basis of plain radiographs it would appear logical to postulate that proximal migration of the humeral head and a diminished subacromial space are a result of a rupture of the RC, although an intact but thinned RC has been seen in many instances. ${ }^{24} 25$ The above mentioned three cases represent Kelly's group III. US failed to depict visible RC tendon substance, and these cases were thus classified as full thickness tears.

In the past 10 years, US and magnetic resonance imaging (MRI) have been introduced into the clinical practice of diagnosing shoulder problems. MRI is superior to US in depicting effusions, ${ }^{26}{ }^{27}$ synovial thickening and pannus tissue formation, ${ }^{4}$ cartilage damage and intraosseus abnormality, ${ }^{48}$ and RC pathology. ${ }^{29}$ However, in comparison with US, MRI is less available, more expensive and time consuming, and less convenient to the patients.

The operator dependency of US and the small number of patients in this series may somewhat limit the generaliseability of our results. We, however, conclude that US is a reliable method in experienced hands for the evaluation of inflammatory changes of the arthritic shoulder. It is to be noted that in advanced stages of RA, the destructive bone changes and tendon ruptures cause upward and medial migration of the humeral head and restricted shoulder motions limiting the usefulness of US in the evaluation of the shoulder in these cases.

This work was supported by the Finnish Cultural Foundation, Helsinki, Finland.

\footnotetext{
1 Riordan J, Dieppe P. Arthritis of the glenohumeral joint. Baillieres Clin Rheumatol 1989;3:607-25.

2 Petersson CJ. Painful shoulders in patients with rheumatoid arthritis. Scand J Rheumatol 1986;15:275-9.

3 Hämäläinen $M$. Epidemiology of upper limb joint affections in rheumatoid arthritis. In: Baumgartner $\mathrm{H}$, Dvorak J, Grob D, Munziger U, Simmen BR, eds. Rheumatoid arthritis. Current trends in diagnostics, conservative treatment, and surgical reconstruction. Stuttgart: George Thieme Verlag, surgical reconstruc

4 Kieft GJ, Dijkmans BAC, Bloem JL, Kroon HM. Magnetic resonance imaging of the shoulder in patients with rheumatoid arthritis. Ann Rheum Dis 1990;49:7-11.
} 
5 van Holsbeeck M, Strouse PJ. Sonography of the shoulder: evaluation of the subacromial-subdeltoid bursa. Am J evaluation of the subacrom

6 Farin PU, Jaroma H, Harju A, Soimakallio S. Shoulder impingement syndrome: sonographic evaluation. Radiology 1990;176:845-9.

7 Middleton WD, Reinus WR, Totty WG, Melson GL. Murphy WA. Ultrasonographic evaluation of the rotator cuff and biceps tendon. J Bone Joint Surg 1986;68A:440-50.

8 Seltzer SE, Finberg HJ, Weissman BN, Kido DK, Collier BD. Arthrosonography: Gray-scale ultrasound evaluation of the shoulder. Radiology 1979;132:467-8.

9 Koski JM. Axillar ultrasound of the glenohumeral joint. J Rheumatol 1989;16:664-7.

10 Hodler J, Terrier B, von Schulthess GK, Fuchs WA. MRI and sonography of the shoulder. Clin Radiol 1991;43:323-7.

11 Arnett FC, Edworthy SM, Bloch DA, McShane DJ, Fries $\mathrm{JF}$, Cooper NS, et al. The American Rheumatism Association 1987 revised criteria for the classification of rheumatoid arthritis. Arthritis Rheum 1988;31:315-23.

12 van der Linden S, Valkenburg HA, Cats A. Evaluation of diagnostic criteria for ankylosing spondylitis. A proposal for modification of the New York Criteria Arthritis Rheum 1984;27:361-8.

13 Gladman DD, Shuckett R, Russel ML, Thorne JC, Schachter RK. Psoriatic arthritis (PSA) - an analysis of 220 patients. Q J Med 1987;62:127-41.

14 Crass JR, Craig EV, Feinberg SB. Hyperextended internal rotation view in rotator cuff ultrasonography. J Clin Ultrasound 1987;15:416-20.

15 Alasaarela EM, Alasaarela ELI. Ultrasound evaluation of painful rheumatoid shoulders. J Rheumatol 1994;21: 1642-8.

16 Bretzke CA, Crass JR, Craig EV, Feinberg SB. Ultrasonography of the rotator cuff. Normal and pathological anatomy. Invest Radiol 1985;20:311-15.

17 Pahle JA, Kvarners L. Shoulder synovectomy. Ann Chir Gynaecol 1985; (suppl) 198:37-9.
18 Middleton WD, Reinus WR, Totty WG, Melson GL, Murphy WA. US of the biceps tendon apparatus. Radiology 1985;157:211-15.

19 Post M, Benca P. Primary tendinitis of the long head of biceps. Clin Orthop 1989;246:117-25.

20 van Holsbeeck MT, Kolowich PA, Eyler WR, Craig JG, Shirazi KK, Habra GK, et al. US depiction of partialthickness tear of the rotator cuff. Radiology 1995;197: 443-6.

21 Farin PU, Jaroma H. Acute traumatic tears of the rotator cuff: Value of sonography. Radiology 1995;197:269-73.

22 Miller CL, Karasick D, Kirtz AB, Fenlin JM. Limited sensitivity of ultrasound for detection of rotator cuff tears. Skeletal Radiol 1989;18:179-83.

23 Brandt TD, Cardone BW, Grant TH, Post M, Weiss CA. Rotator cuff sonography: a reassessment. Radiology 1989;173:323-7.

24 Kelly IG. Surgery of the rheumatoid shoulder. Ann Rheum Dis 1990;49:824-9.

25 Cruess RL. Rheumatoid arthritis of the shoulder. Orthop Clin North Am 1980;11:333-42.

26 Hollister MS, Mack LA, Patten RM, Winter TC III, Matsen FA III, Veith RR. Association of sonographically detected subacromial/subdeltoid bursal effusion and intraarticular fluid with rotator cuff tear. Am J Roentgenol 1995;165: 605-8.

27 Schweitzer ME, Magbalon MJ, Fenlin JM, Frieman BG, Ehrlich S, Epstein RE. Effusion criteria and clinical importance of glenohumeral joint fluid: MR imaging evaluation. Radiology 1995;194:821-4.

28 Beltran J, Caudill JL, Herman LA, Kantor SM, Hudson PN, Noto AM, et al. Rheumatoid arthritis: MR imaging manifestations. Radiology 1987;165:153-7.

29 Iannotti JP, Zlatkin MB, Esterhai JL, Kressel HY, Dalinka MK, Spindler KP Magnetic resonance imaging of the shoulder. Sensitivity, specificity, and predictive value. J Bone Joint Surg 1991;73A:17-29. 